\title{
SpeciesPrimer: A bioinformatics pipeline dedicated to the design of qPCR primers for the quantification of bacterial species
}

\author{
Matthias Dreier ${ }^{\text {Corresp., } 1,2}{ }$, Hélène Berthoud ${ }^{1}$, Noam Shani ${ }^{1}$, Daniel Wechsler ${ }^{1}$, Pilar Junier ${ }^{2}$ \\ ${ }^{1}$ Agroscope, Bern, Switzerland \\ 2 \\ Laboratory of Microbiology, University of Neuchâtel, Neuchâtel, Switzerland \\ Corresponding Author: Matthias Dreier \\ Email address: matthias.dreier@agroscope.admin.ch
}

Background. Quantitative real-time PCR (qPCR) is a well-established method for detecting and quantifying bacteria, and it is progressively replacing culture-based diagnostic methods in food microbiology. High-throughput qPCR using microfluidics brings further advantages by providing faster results, decreasing the costs per sample and reducing errors due to automatic distribution of samples and reagents. In order to develop a high-throughput qPCR approach for the rapid and cost-efficient quantification of microbial species in complex systems such as fermented foods (for instance, cheese), the preliminary setup of qPCR assays working efficiently under identical PCR conditions is required. Identification of target-specific nucleotide sequences and design of specific primers are the most challenging steps in this process. To date, most available tools for primer design require either laborious manual manipulation or high-performance computing systems.

Results. We developed the SpeciesPrimer pipeline for automated high-throughput screening of speciesspecific target regions and the design of dedicated primers. Using SpeciesPrimer specific primers were designed for four bacterial species of importance in cheese quality control, namely Enterococcus faecium, Enterococcus faecalis, Pediococcus acidilactici and Pediococcus pentosaceus. Selected primers were first evaluated in silico and subsequently in vitro using DNA from pure cultures of a variety of strains found in dairy products. Specific qPCR assays were developed and validated, satisfying the criteria of inclusivity, exclusivity and amplification efficiencies.

Conclusion. In this work, we present the SpeciesPrimer pipeline, a tool to design species-specific primers for the detection and quantification of bacterial species. We use SpeciesPrimer to design qPCR assays for four bacterial species and describe a workflow to evaluate the designed primers.

SpeciesPrimer facilitates efficient primer design for species-specific quantification, paving the way for a fast and accurate quantitative investigation of microbial communities. 


\section{SpeciesPrimer: A bioinformatics pipeline dedicated to the}

\section{3 design of qPCR primers for the quantification of bacterial}

\section{4 species}

5

6

Matthias Dreier ${ }^{1,2}$, Hélène Berthoud ${ }^{1}$, Noam Shani ${ }^{1}$, Daniel Wechsler ${ }^{1}$, Pilar Junier ${ }^{2}$

${ }^{1}$ Agroscope, Schwarzenburgstrasse 161, CH-3003 Bern, Switzerland

${ }^{2}$ Laboratory of Microbiology, University of Neuchâtel, Emile-Argand 11, CH-2000 Neuchâtel, Switzerland

\section{Corresponding Author:}

Matthias Dreier ${ }^{1,2}$

Schwarzenburgstrasse 161, Bern, CH-3003, Switzerland

Email address: matthias.dreier@agroscope.admin.ch

\section{Abstract}

Background. Quantitative real-time PCR (qPCR) is a well-established method for detecting and quantifying bacteria, and it is progressively replacing culture-based diagnostic methods in food microbiology. High-throughput qPCR using microfluidics brings further advantages by providing faster results, decreasing the costs per sample and reducing errors due to automatic distribution of samples and reagents. In order to develop a high-throughput qPCR approach for the rapid and cost-efficient quantification of microbial species in complex systems such as fermented foods (for instance, cheese), the preliminary setup of qPCR assays working efficiently under identical PCR conditions is required. Identification of target-specific nucleotide sequences and design of specific primers are the most challenging steps in this process. To date, most available tools for primer design require either laborious manual manipulation or highperformance computing systems.

Results. We developed the SpeciesPrimer pipeline for automated high-throughput screening of species-specific target regions and the design of dedicated primers. Using SpeciesPrimer, 
32 specific primers were designed for four bacterial species of importance in cheese quality control,

33 namely Enterococcus faecium, Enterococcus faecalis, Pediococcus acidilactici and

34 Pediococcus pentosaceus. Selected primers were first evaluated in silico and subsequently in

35 vitro using DNA from pure cultures of a variety of strains found in dairy products. Specific

36 qPCR assays were developed and validated, satisfying the criteria of inclusivity, exclusivity and

37 amplification efficiencies.

38 Conclusion. In this work, we present the SpeciesPrimer pipeline, a tool to design species-

39 specific primers for the detection and quantification of bacterial species. We use SpeciesPrimer

40 to design qPCR assays for four bacterial species and describe a workflow to evaluate the

41 designed primers. SpeciesPrimer facilitates efficient primer design for species-specific

42 quantification, paving the way for a fast and accurate quantitative investigation of microbial

43 communities.

\section{Introduction}

45 Quantitative real-time PCR (qPCR) is a well-established method for the detection and

46 quantification of bacteria in microbiology, for instance in the context of pathogen detection in

47 clinical and veterinary diagnostics and food safety (Cremonesi et al. 2014; Curran et al. 2007;

48 Garrido-Maestu et al. 2018; Ramirez et al. 2009). Culture-based diagnostic methods are

49 progressively being replaced by qPCR due to advantages such as faster results, more specific

50 detection, and the ability to detect sub-dominant populations (Postollec et al. 2011). High-

51 throughput microfluidic qPCR brings further advantages including the fast generation of results,

52 a lower cost per sample and fewer errors due to automatic distribution of samples and reagents.

53 However, in order to work efficiently high-throughput qPCR systems use identical PCR

54 chemistry and PCR conditions for all reactions taking place on a single chip. Therefore, existing qPCR assays are often not suitable and new primers have to be designed (Hermann-Bank et al.

56 2013; Ishii et al. 2013; Kleyer et al. 2017).

57 The main challenges for the successful development of any qPCR assay are the identification of a specific target nucleotide sequence and the design of primers that bind exclusively to that target

59 sequence. Before microbial draft genomes became widely available, the 16S rRNA gene

60 sequence was frequently used as a target sequence. However, the regions that are targeted in the

61 16S rRNA gene do not provide sufficient resolution to differentiate between closely related

62 bacterial species (Moyaert et al. 2008; Torriani et al. 2001; Wang et al. 2007). Further, 
63 housekeeping genes such as, for instance, $t u f$, rec $A$ and $p h e S$, were successfully used as target

64 sequences for a variety of bacterial species in fermented foods (Falentin et al. 2010; Masco et al.

65 2007; Scheirlinck et al. 2009). Today, the steadily increasing number of prokaryotic draft

66 genomes facilitates the identification of new and unique target regions. This, in combination with

67 the increased computing power, makes it now possible to screen and compare hundreds of genomes and to predict unique target sequences in a relatively short time.

Various commercial and open source programs facilitate the design of specific primers for a target sequence, such as the standard tools Primer3 and Primer-BLAST (Untergasser et al. 2012; Ye et al. 2012). Primer3 predicts suitable PCR primers for an input target sequence, while Primer-BLAST combines Primer3 with a BLAST search in a selected nucleotide sequence database to assess the specificity of the primers for the target sequence. Table 1 provides an overview of the features of different primer design tools and pipelines. PrimerMiner (Elbrecht et al. 2017) is a tool that automatically downloads sequences of marker genes for taxonomic group specified by the user and creates alignments and consensus sequences as target sequences for the design of degenerate primers. PrimerServer (Zhu et al. 2017) allows to design primers for multiple sites across a whole genome sequence and performs a specificity check. Tools and pipelines that encompass both the identification of target sequences from bacterial draft genomes and the design of primer candidates include, for instance, RUCS, the find_differential_primers (fdp) pipeline and TOPSI (Pritchard et al. 2012; Thomsen et al. 2017; Vijaya Satya et al. 2010). RUCS is able to identify unique core sequences in a positive set of genomes (target) compared to a negative set of genomes (non-target). It designs primers for the core sequences and validates them with an in silico PCR validation method against the positive and negative reference sets. Similarly, the fdp pipeline designs primers for a set of positive genomes and, further, allows to extract primers specific to subclasses of the positive set and performs specificity check against a negative set of genomes. TOPSI is an automated high-throughput pipeline for the design of primers, primarily developed for pathogen-diagnostic assays. It identifies sequences present in 89 all input genomes and designs specific primers accordingly.

90 We aimed to design a series of primers that function with the same qPCR cycling conditions and 91 primer concentrations for later usage in a high-throughput microfluidic qPCR platform. RUCS, 92 fdp and TOPSI can be used to design species-specific primers and offer high-throughput primer design. However, TOPSI could not be used because no Linux-based cluster was available. RUCS 
94 and fdp were not able to design primers for all our target species. Therefore, these pipelines were 95 not suitable for our high-throughput approach.

96 This study presents a new pipeline named SpeciesPrimer developed for automated high-

97 throughput screening for species-specific target regions combined with the design of primer

98 candidates for these sequences. The process of primer design is fully automated from the 99 download of bacterial genomes to the quality control of primer candidates. The pipeline runs on

100 a standard computer with a multi-core processor and a minimum of 16 GB RAM. We have

101 applied the SpeciesPrimer pipeline to a set of four bacterial species occurring in cheese and other 102 dairy products and validated the primers in silico and in vitro by performing qPCR experiments 103 with a variety of target and non-target strains.

\section{Description}

\section{Overview}

106 The SpeciesPrimer pipeline consists of three main parts (Table 2). First, genome assemblies are 107 downloaded, annotated and then subjected to quality control. Second, a pan-genome analysis is 108 performed to identify single copy core genes. Conserved sequences of these core genes are then 109 extracted and the specificity for the target species is assessed. Finally, primers are designed for

110 these species-specific conserved core gene sequences and subsequently evaluated in a primer 111 quality control step. An overview of the features of the tools used for SpeciesPrimer can be 112 found in Table S1.

\section{Part 1: Input genome assemblies}

114 The minimal command line input for the pipeline is the species name. Further, a list of non-target 115 species names can be specified (e.g., species found in the investigated ecosystem but that should 116 not be detected in the specific qPCR assay). For downloading genome assemblies from the 117 National Center for Biotechnology Information (NCBI) automatically, a valid e-mail address is 118 required for accessing the NCBI E-utilities services (Sayers 2009). The pipeline works with a 119 pre-formatted NCBI BLAST database (nt), containing partially non-redundant nucleotide 120 sequences. A local copy of the nt database is required. It can be downloaded from NCBI using 121 the update_blastdb.pl script from the BLAST+ package (Altschul et al. 1990), via FTP from the 122 NCBI FTP server or with the pipeline script (getblastdb.py). The nt database, which consists of 123 sequences from GenBank, EMBL (European Molecular Biology Laboratory) and DDBJ (DNA 
124 Data Bank of Japan), was selected because it has a large coverage of diverse sequences, but it is

125 not as large as for example the refseq genomic database (Tao et al. 2011). The evaluation of the

126 specificity of the target sequence for the target species does not rely on small differences in the

127 nucleotide sequence, but on the overall similarity. Therefore, even with one genome sequence

128 per non-target species we would expect to find similarities in the core genes of the non-target

129 species. Each additional genome of this species in a database would then allow finding more

130 potential sequence similarities in shell genes, cloud genes and strain-specific genes. On the one

131 hand, a more extensive database could better predict the specificity of a sequence, but on the

132 other it would increase the size of the database and the time required for the BLAST search.

133 The user-provided species name is used to search for genome assemblies in the NCBI database.

134 The Biopython Entrez module (Cock et al. 2009) searches the NCBI taxonomic identity (taxid)

135 for the target species in the taxonomy database and downloads the genome assembly summary

136 report. Afterwards, SpeciesPrimer downloads the genome assemblies in FASTA format from the

137 NCBI RefSeq FTP server using the links specified in the summary report. Finally, the

138 downloaded genome assemblies are annotated with Prokka (Seemann 2014).

139 The quality of the genome assemblies is a crucial factor for the pan-genome analysis. Genome

140 assemblies deposited with the wrong taxonomic label or low-quality assemblies drastically

141 reduce the number of identified core genes and of conserved sequences for primer design. The

142 initial quality control step is intended to remove such assemblies from the subsequent analysis.

143 For the verification of the taxonomic classification, the user can choose one or several genes

144 from five conserved housekeeping genes (16S rRNA, tuf, recA, dnaK and pheS). Genome

145 assemblies without an annotation for the specified conserved housekeeping genes or genome

146 assemblies consisting of more than 500 contigs are removed from the downstream pan-genome

147 analysis. The sequences of the specified conserved housekeeping genes are blasted against the

148 local nt database. Genome assemblies pass the quality control if the best BLAST hit for all

149 sequences is a sequence arising from the target species.

\section{Part 2: Identification of target sequences for primer design}

151 A pan-genome analysis is performed using Roary (Page et al. 2015) to identify the core genes of

152 the target species. Based on the results of the pan-genome analysis, single copy core genes are

153 identified. The gene_presence_absence.csv produced by Roary reports the presence (or absence)

154 of every annotated gene for every input genome assembly. Single copy core genes are the genes 
155 for which the number of assemblies harboring the sequence and the number of total identified

156 sequences equals the number of total input assemblies. An sqlite3 database containing all

157 annotated sequences of all assemblies is compiled using the DBGenerator.py script from the

158 Microbial Genomics Lab GitHub repository (https://github.com/microgenomics/tutorials). This

159 database is queried for single copy core genes and the nucleotide sequences are saved in multi-

160 FASTA format. Each multi-FASTA file contains the sequences of one single copy core gene

161 from each input genome assembly. These sequences are aligned using the probabilistic multiple

162 alignment program Prank (Löytynoja 2014). A consensus sequence with ambiguous bases is then

163 created using the consambig function from the EMBOSS package (Rice et al. 2000). The

164 alignments and extraction of the consensus sequences are performed in parallel for several core

165 genes using GNU parallel (Tange 2011). Continuous consensus sequences longer than the

166 minimal PCR product length, harboring less than two ambiguous bases in the range of 20 bases

167 are used for the subsequent steps of the pipeline.

168 These conserved consensus sequences are used for a BLAST search against the local nt database

169 using the discontiguous BLAST algorithm and an e-value cutoff of 500. For all hits in the

170 BLAST results, the species name is extracted from the sequence description and compared with

171 the names in the species list (non-target species). If any species name in the species list matches

172 a hit in the BLAST results the corresponding query sequence is discarded, otherwise the

173 sequence is classified as specific for the target and considered for primer design.

\section{Part 3: Primer design}

175 Primer3 is used to design primers for the unique single copy core gene sequences. As pipeline

176 default the optimum primer melting temperature is set to $60^{\circ} \mathrm{C}$ and the maximal primer length is

177 set to 26 bases, all other settings are the default settings of the primer3web version

178 (http://primer3.ut.ee, accessed November 29, 2018). The minimal and maximal amplicon size of

179 the PCR product can be specified individually for every target species through the command line

180 options. The other parameters for primer3 cannot be changed individually, but the general

181 primer3 settings can be changed by modifying the primer3 settings file.

182 The primer quality control consists of three parts, an in silico PCR to evaluate the specificity of

183 the primer for the template, an estimation of secondary structures of the amplicon sequence and

184 the estimation of the potential to form primer dimers. The specificity check (Figure 1) for each

185 primer pair is performed with MFEprimer-2.0 (Qu et al. 2012). For the evaluation of the 
186 specificity, three indexed databases are generated: the target template database, the non-target 187 sequence database and the target genome database. The target template database consists of the 188 unique conserved core gene sequences used as template for primer design. The non-target

189 sequence database is compiled from sequences of non-target species that show similarities to the 190 primer sequences. To identify these sequences, a BLAST search with all primers against the 191 local nt database is performed. BLAST hits with a species name in the description matching a 192 name in the user-specified non-target species list are selected. These selected sequences and 1934000 base pairs up- and downstream are extracted from the nt database using the blastdbcmd 194 tool. The target genome database is composed of maximal 10 of the input genome assemblies. If 195 the assembly summary report from the automatic download of genome assemblies from NCBI is available, the genome assemblies as complete as possible are preferred (assembly status: complete $>$ chromosome $>$ scaffold $>$ contig). The target sequence database is used to evaluate the maximum primer pair coverage (PPC, maximum value $=100)$, a value used by MFEprimer2.0 to score the ability of the primer pair to bind to a DNA template. All primer pairs with a PPC value lower than the specified threshold (mfethreshold, default $=90)$ for their template are excluded. Next, MFEprimer-2.0 is used to score the binding of the primer pairs to the sequences of the non-target sequence and the target genome database. The difference of the PPC for the DNA template and the specified threshold $(\Delta$ threshold $=$ PPC - mfethreshold $)$ is used as a threshold for the maximum PPC value a primer pair is allowed to have for a non-target sequence. Strong secondary structures at the 5'- or the 3'- end of the PCR product could impair efficient primer binding. Therefore, the PCR products of the primer pairs are submitted to mfold (Zuker et al. 1999) to exclude PCR products with strong secondary structures at the annealing temperature of $60{ }^{\circ} \mathrm{C}$. Moreover, as primer dimers can yield unspecific signals during the qPCR run, the 3'ends of the primer pairs are checked for their potential to form homo- or hetero-dimers using a Perl script (MPprimer_dimer_check.pl) from MPprimer (Shen et al. 2010).

211 The pipeline output is a list containing the primer name, primer pair coverage (MFEprimer) and 212 penalty values, primer and template sequences and melting temperatures (Primer3). Further, a 213 report of the genome assembly quality control, a file containing the pipeline run statistics, the 214 core gene alignment and the phylogeny in newick format can be found in the output directory. 


\section{Materials \& Methods}

\section{Primer design}

217 SpeciesPrimer pipeline runs were performed on a virtual machine (Oracle VM VirtualBox 5.2.8)

218 with Ubuntu 16.04 (64-bit) and Docker installed, using 22 of 24 logical processors from two

219 Intel Xeon E5-2643 CPUs, 32 GB of RAM, a solid-state drive and a LAN Internet connection.

220 To show the performance of the SpeciesPrimer pipeline on consumer hardware the runs were

221 repeated on a laptop with an Ubuntu 16.04 (64-bit) operating system, an i7-3610QM CPU (8

222 logical processors), 8 GB RAM, a solid-state drive and a wireless LAN Internet connection. The

223 used Docker image is available at https://hub.docker.com/r/biologger/speciesprimer.

224 The species list consisted of 259 species and subspecies names detected in dairy products,

225 namely from species names collected from data of 16S rRNA meta-genome sequencing studies

226 in milk and cheese varieties (Marco Meola Agroscope, pers. comm.) and dairy-related bacteria

227 from the list of bacterial species and subspecies with technological beneficial use in food

228 products (Almeida et al. 2014).

229 The SpeciesPrimer pipeline was run with the input genome assemblies, parameters and the

230 species list specified in the supplemental information (Dataset S1). Genome assemblies from the

231 Agroscope Culture Collection were included for the Pediococci.

\section{In silico validation}

233 For the in silico validation, PCR products for the designed primer pairs were used for an online

234 BLAST search against the RefSeq Genomes Database (refseq_genomes) limited to bacterial

235 genomes. The search was performed by qblast (Biopython), using blastn, the maximum hitlist

236 size was set to 5000 and the expect threshold (e-value) was set to 500.

237 Primer pairs were tested for specificity using online Primer-BLAST. The primers were blasted

238 against the nucleotide collection BLAST database (nr) limited to sequences from bacteria. The $\mathrm{nr}$

239 (non-redundant nucleotide) database was chosen to get the broadest coverage for the BLAST

240 search. Default settings were used, except for the primer specificity stringency that was set to

241 ignore targets that have nine or more mismatches to the primer. 


\section{In vitro validation}

243 The inclusivity of the primer pairs was assayed by performing qPCR with 2 ng DNA of 21 to 25

244 strains of the target species in technical duplicates. The PCR efficiency was examined by ten-

245 fold dilution series of the type strain DNA in a range from $10^{6}$ to $10^{1}$ genome copies per reaction.

246 DNA concentration for the corresponding number of genome copies was estimated by taking the

247 genome size of the type strain (https://www.ncbi.nlm.nih.gov/genome) and an average weight of

$2481.096 \cdot 10^{-21} \mathrm{~g}$ per base pair.

249 The exclusivity of the primer pairs was assayed by performing qPCR in technical duplicates with

$2502 \mathrm{ng}$ DNA from various bacterial species found in dairy products. Because the number of

251 samples per run was limited, four separate runs were required to measure all non-target strains.

252 In each run three strains of the target species (positive control) and a no template control were

253 included.

\section{Bacterial strains}

255 Strains stored within the Agroscope Culture Collection at $-80{ }^{\circ} \mathrm{C}$ in sterile reconstituted skim

256 milk powder $(10 \%, \mathrm{w} / \mathrm{v})$, were reactivated and cultivated according to the conditions specified

257 in Dataset S2.

\section{DNA extraction}

259 Unless otherwise noted, all reagents were purchased from Merck, Darmstadt, Germany.

260 Bacterial pellets harvested from $1 \mathrm{ml}$ culture by centrifugation (10000 x g, $5 \mathrm{~min}$, room

261 temperature) were used for DNA extraction. For a pre-lysis treatment, the bacterial cells were

262 incubated in $1 \mathrm{ml}$ of $50 \mathrm{mM}$ sodium hydroxide for $15 \mathrm{~min}$ at room temperature. Afterwards cells

263 were collected by centrifugation ( $10000 \times \mathrm{g}, 5 \mathrm{~min}$, room temperature) and then treated with

264 lysozyme $(2.5 \mathrm{mg} / \mathrm{ml}$ dissolved in $100 \mathrm{mM}$ Tris(hydroxymethyl)aminomethane, $10 \mathrm{mM}$

265 ethylendiaminetetraacetic acid (EDTA; Calbiochem, San Diego, USA), 25 \% (w/v) sucrose, pH

266 8.0) for 1 hour at $37^{\circ} \mathrm{C}$. After the pre-lysis treatment, the bacterial cells were collected by

267 centrifugation (10000 x g, $5 \mathrm{~min}$, room temperature). Cell lysis and genomic DNA extraction

268 was performed using the EZ1 DNA Tissue kit and a BioRobot ${ }^{\circledR}$ EZ1 workstation (Qiagen,

269 Hilden, Germany) according to the manufacturer's instructions and eluted in a volume of $100 \mu 1$.

270 The DNA concentration was measured using a NanoDrop ${ }^{\circledR}$ ND-1000 Spectrophotometer

271 (NanoDrop Technologies, Thermo Fisher Scientific, Waltham, MA, USA). 


\section{Quantitative real-time PCR}

273 The qPCR assays were performed in a total reaction mix volume of $12 \mu 1$ containing $6 \mu 12 \mathrm{x}$

274 SsoFast ${ }^{\mathrm{TM}}$ EvaGreen ${ }^{\circledR}$ Supermix with low ROX (Biorad, Cressier, Switzerland), $500 \mathrm{nM}$ of

275 forward and reverse primers, respectively, and $2 \mu 1$ of DNA. Each sample was measured in

276 technical duplicates. The qPCR cycling conditions were an initial denaturation at $95^{\circ} \mathrm{C}$ for 1

277 minute followed by 35 cycles of $95^{\circ} \mathrm{C}$ for 5 seconds and $60{ }^{\circ} \mathrm{C}$ for 1 minute. For the melting

278 curve analysis, a gradient from $60-95^{\circ} \mathrm{C}$ with $1{ }^{\circ} \mathrm{C}$ steps per 3 seconds was performed. All

279 qPCR assays were run on a Corbett Rotor-Gene 3000 (Qiagen). The analysis was performed

280 using Rotor-Gene 6000 Software 1.7 with dynamic tube normalization and a threshold of 0.05

281 for quantification cycle $(\mathrm{Cq})$ value calculation, the five first cycles were ignored for the

282 determination of the $\mathrm{Cq}$ values. The peak calling threshold for the melt curve analysis was set to

$283-2 \mathrm{dF} / \mathrm{dT}$ and a temperature threshold was set $2{ }^{\circ} \mathrm{C}$ lower than the positive control peak.

\section{Phylogeny and average nucleotide identity calculations}

285 The phylogeny was created with Roary and FastTree 2 during the pipeline runs and iTOL

286 (Letunic \& Bork 2019) was used to visualize the tree. Average nucleotide identity (ANI)

287 calculations were performed with pyani v0.2.9 (Pritchard et al. 2016) using the ANIm method.

288 The heatmap was created from the ANIm_percentage_identity.tab output file using the

289 clustermap function of the python seaborn module and modified color bar settings from pyani.

290 For the color bars on top and on the left of the heatmap, the assemblies were assigned to the

291 same color as in the phylogeny tree. Row and column names (genome assembly accessions) can

292 be found in Dataset S3.

\section{Comparison of primer design pipelines}

294 The positive genome sets for RUCS and fdp were the same genome assemblies used for the

295 SpeciesPrimer pipeline. SpeciesPrimer uses by default the NCBI nt database and the species list

296 for the specificity checks, whereas RUCS and fdp require a negative set of genomes. Therefore, a

297 set of (representative) genome assemblies from NCBI was downloaded for the species from the

298 species list. From these assemblies a BLAST database was prepared for SpeciesPrimer. The

299 same genome assemblies, excluding the assembly of the target species, were used as a negative

300 set for RUCS and to make a BLAST database for fdp. For both tools, the minimal and maximal

301 PCR product size was set to 70 and 200, respectively. The tab separated config file for fdp was 
302 created using the assembly accession as name, the species as class and providing the absolute

303 path of the genome assembly files. The script was started with the blastdb option to provide the

304 path to the previously prepared BLAST database with the non-target genome sequences. For

305 RUCS the entry point full was selected and the annotation of the target sequences was omitted.

306 SpeciesPrimer was configured to run with the custom BLAST database, without a species list

307 and the download and annotation step for the genome assemblies was omitted to provide

308 comparable running conditions. The accessions of the input genome assemblies and the

309 commands used can be found in Dataset S4. Primers used for a specificity check using Primer-

310 BLAST (nr database limited to sequences from bacteria) were the two primer pairs with the best

311 score in the results_best.tsv files (RUCS), the two best ranked primer pairs for SpeciesPrimer

312 and the primers reported in the universal_primers.eprimer3 files (fdp).

\section{Results}

\section{Primer design}

315 The SpeciesPrimer pipeline runs were completed in two to eight hours, excluding the time

316 required for downloading and annotation of the genome assemblies. Depending on the number of

317 genome assemblies, downloading and annotation of the genome assemblies took from 24

318 minutes (27) to 12 hours 27 minutes (575). The average time for downloading and annotation of

319 single assemblies was two seconds and one minute six seconds, respectively. On the consumer

320 laptop using a wireless LAN Internet connection the time required for the downloads has

321 doubled, while the annotation took 1.8 times longer. The pipeline runs took in total three times

322 longer, they were completed in seven to 29 hours. The analysis of the Enterococcus faecalis,

323 Enterococcus faecium, Pediococcus acidilactici and Pediococcus pentosaceus assemblies

324 resulted in 15, 2, 2 and 160 identified primer pair candidates, respectively (Table 3). The primer

325 pair candidates for E. faecalis and P. pentosaceus were filtered for the highest primer pair

326 coverage score (E. faecalis: 2; P. pentosaceus: 29); for P. pentosaceus, only the two primer pairs

327 with the lowest primer pair penalty values were selected.

328 The phylogeny tree from the concatenated core genes of E. faecium shows the phylogenetic

329 distance of two distinct groups of sequences, a main cluster with 531 sequences and a subcluster

330 with 44 sequences (Figure 2). The tree made with the concatenated core gene sequences of $P$.

331 acidilactici shows the phylogenetic distance of one sequence from all other sequences (Figure 3). 
332 From this observation, the existence of different taxonomic units was suspected. Calculation of

333 the average nucleotide identity (ANI) has been proposed as a valuable tool to determine species

334 boundaries (Richter \& Rossello-Mora 2009). Therefore, we performed ANI calculations for the

335 genome assemblies and displayed the results in a clustered heatmap (Figure 4). All genome

336 assemblies show an alignment coverage of at least $60 \%$ to each other (Dataset S3), indicating

337 they are correctly assigned at the genus level. The clustering of the E. faecium genome

338 assemblies in Figure 4 A shows two distinct clusters corresponding to the clusters in the

339 phylogenetic tree (Figure 2). The assemblies of the two clusters have ANIm values at the border

340 of the species threshold cutoff as depicted by the white to light purple colored cells. Clustering of

341 the $P$. acidilactici genome assemblies in Figure $4 \mathrm{~B}$ shows three distinct clusters corresponding

342 to the clusters in the phylogenetic tree (Figure 3). The purple cells indicate that the assemblies of

343 two larger clusters belong to the same species, while the assembly with the orange color bar has

344 ANIm percentage identity values below the proposed species threshold cutoffs (95 - 96 \%) (Kim

345 et al. 2014; Richter \& Rossello-Mora 2009) as indicated by the blue cells. P. acidilactici strain

346 FAM 18987 should therefore probably be assigned to a new species or subspecies. However, for

347 certain species lower boundary cutoffs might be reasonable (Ciufo et al. 2018). According to the

348 current taxonomic classification, we proceeded with the assumption that these genome

349 assemblies reflected the actual diversity of strains and thus included the assemblies for the

350 primer design.

351 Two test cases were generated to exemplify the influence of the input genome assemblies on the

352 pipeline results. Firstly, a single genome assembly with a wrong taxonomic label was used as

353 input in addition to the correctly labelled genome assemblies. Introducing a genome assembly

354 with a wrong taxonomic label (GCF_000415325.2, E. faecalis) into the pool of E. faecium

355 genome assemblies resulted in a decrease of identified core genes (from 1131 to 43) and

356 provided no species-specific sequence. Secondly, the genome assembly of the $P$. acidilactici

357 strain (FAM 18987) that was distinct from the other assemblies in the phylogenetic tree and had

358 ANI values below the species threshold cutoff was excluded from the pipeline run. This resulted

359 in an increased number of identified core genes (from 921 to 1238), of species-specific

360 sequences (from 54 to 516) and of reported primer pairs (from 2 to 53). 


\section{$361 \quad$ In silico validation}

362 Two parameters were selected as criteria for the primer validation using web-based BLAST.

363 First, the BLAST hits for the predicted PCR product sequence should only match the target

364 species. If sequences of other bacterial species matched to parts of the sequence, the

365 corresponding primer pairs were discarded, unless more than three mismatches were found in

366 each primer-binding region for the forward and reverse primers. Second, the primer binding sites

367 in the target sequences were not allowed to have mismatches in the 3 '-end region. The criterion

368 for the primer validation by Primer-BLAST was that no predicted PCR products for other

369 bacterial species had been reported by Primer-BLAST. Primer pairs exclusively binding to the

370 target sequence of the target species were classified as specific. The results of the in silico

371 validation are summarized in Table 4. With the exception of Ec_faeca_g3060_1_P0 and

372 Ec_faeci_cysS_3_P1, all primer pairs showed a perfect match to their target sequences. For

373 primer pair Ec_faeca_g3060_1_P0, the first three nucleotides of one sequence out of 690 are

374 missing in the forward primer-binding region. For Ec_faeci_cysS_3_P1, only one sequence out

375 of 1058 aligned sequences showed a single nucleotide transition in the reverse primer-binding

376 region (Dataset S5, page 2-3).

\section{In vitro validation}

378 The specificity of the qPCR assays was assessed with 21 to 25 strains of the target species

379 (inclusivity) and 120 non-target bacterial strains found in dairy products (exclusivity). The qPCR

380 assay performance was assessed by 10-fold dilution series of type strain DNA from $10^{6}$ to $10^{1}$

381 copies per reaction. The results of the qPCR runs were interpreted as positive if both qPCR

382 reactions (duplicates) reached the fluorescent threshold before quantification cycle 35 and the

383 peak of the melting curve analysis was above the peak calling threshold $(-2 \mathrm{dF} / \mathrm{dT})$. A summary

384 of the results is shown in Table 5. The primer sequences can be found in Table S2. The

385 inclusivity of the qPCR assays was $100 \%$ for the assays Ec_faeca_acuI, Ec_faeca_g3060,

386 Ec_faeci_cysS, Pd_acidi_asnS, Pd_acidi_g1164, Pd_pento_nagK and Pd_pento_g4364. Only

387 one qPCR assay, Ec_faeca_purD was negative for one of the tested target strains.

388 Out of the 120 non-target strains analyzed to determine the exclusivity of the qPCR assays

389 (Figure 5), all strains were negative for Ec_faeca_acuI and Pd_acidi_asnS. The assay

390 Pd_pento_nagK targeting P.pentosaceus was positive for two out of three tested Leuconostoc 
391 lactis strains, the fluorescence signal reached the threshold after $\mathrm{Cq} 26$, and the melting curve

392 analysis showed a peak at $85^{\circ} \mathrm{C}$, while the positive control samples for this assay displayed a

393 peak at $83.5^{\circ} \mathrm{C}$. Nine out of the 120 non-target strains were positive for the Ec_faeca_g3060

394 qPCR assay, for these samples the fluorescence signals reached the threshold after Cq 26 and had

395 a melting curve peak at a higher temperature than the target PCR product. The assays

396 Pd_acidi_g1164 and Pd_pento_g4364 were positive for five and eight non-target strains,

397 respectively. Notably, all three tested Lactobacillus paracasei strains were positive for the

398 Pd_acidi_g1164 assay, the fluorescence signal reached the threshold around Cq 21 and 22 and

399 they showed a distinct melting curve peak at $86^{\circ} \mathrm{C}$.

400 The calculated efficiency of the qPCR assays was between 92 and $100 \%$. The linear regression

401 equations $(\mathrm{C} q=$ slope $* \log ($ copies $)+$ intercept $)$ had slopes between -3.329 and -3.523 and

402 correlation coefficients of 0.990 or above. Dataset S6 contains the qPCR raw data and Dataset S7

403 a summary of the qPCR data.

\section{Comparison of primer design pipelines}

405 The running times and the number of reported primer pairs of RUCS, the fdp pipeline, and

406 SpeciesPrimer were compared. The download and annotation times were not considered since

407 RUCS and fdp do not include this feature. RUCS and fdp were both able to design primer pairs

408 for all four bacterial targets. The runs with RUCS were completed in two hours and 11 minutes

409 to five hours and 20 minutes and between 107 and 629 primer candidates were reported. The

410 specificity check using online Primer-BLAST showed that the best-ranked primer pair for each

411 of the targets was specific and perfectly matched to the primer binding region for all targets in

412 the nr database. The fdp runs were completed in 2 minutes to 17 hours 44 minutes. Three primer

413 pairs were reported for E. faecium and $P$. acidilactici and six primer pairs were reported for

414 E. faecalis and P. pentosaceus. Primer-BLAST results indicate that the best primer pairs for all

415 target species are specific. The best primer pair for P. acidilactici showed a two-nucleotide

416 mismatch in the primer binding region of one target sequence. A one-nucleotide mismatch in the

417 primer binding region of one target sequence was also observed in the primer pair for $E$. faecalis

418 (Dataset S4, Primer-BLAST summary). The results of the SpeciesPrimer runs differ from the

419 runs with the nt BLAST database presented in detail above. For the Enterococci the best reported

420 primer pairs remain the same, while different primer pairs were ranked best for the Pediococci. 
421 In summary, all pipelines were able to design species-specific primers for all of the four target

422 species using the given input sequences. The results of the comparison are summarized in Table 4236.

\section{Discussion}

425 After setup of the SpeciesPrimer Docker container, the download of the local BLAST database

426 and the selection of the SpeciesPrimer run settings, no further manual handling was required to

427 get primer pair candidates for all four bacterial species after a total time of 44 hours and 30

428 minutes (high end desktop). The number of input genomes and subsequently the number of

429 retrieved primer pairs for the specificity check have the highest impact on speed. During the

430 specificity check, blasting the primer sequences optimized for short sequences (blastn-short) and

431 the subsequent compilation and indexing of the non-target sequence database are the most time

432 consuming steps.

433 The results of the SpeciesPrimer pipeline for the four target species ranged from two to 160

434 identified primer pair candidates. Several factors can influence the number of identified primer

435 pairs, such as the quality of the input genome assemblies, assemblies with wrong taxonomic

436 labels and the genetic diversity within the species. A low-quality assembly with missing

437 sequences or contaminations can decrease the number of identified core genes. The initial quality

438 control helps to minimize the risk that such assemblies are included in the pipeline runs.

439 However, also an increased sequence diversity, either due to sequencing errors, assembly errors

440 or real diversity, limits the number and the length of identified conserved sequences.

441 Subsequently this affects the yield of reported primer pairs, since the pipeline selects highly

442 conserved sequences for primer design. The two test cases designed to exemplify the influence

443 of the input genome assemblies on the pipeline results illustrate that the SpeciesPrimer pipeline

444 performs best on closely related (same species) genome assemblies with a good overall quality.

445 The specificity of the designed primers was evaluated in silico by BLAST with a more extensive 446 database (RefSeq Genome) than the one used for the specificity check during primer design. The

447 validation showed that the specificity of the tested amplicons was high and no other species than

448 the target species had an identical sequence. Most target sequences in the database showed a

449 perfect match for the primers in the primer-binding region. For all tested primer pairs, only the

450 expected PCR products for the target species and no amplicons for other sequences were

451 predicted by Primer-BLAST. The results of Primer-BLAST indicate that the reported primer 
452 pairs were very specific, even though the species list used for the specificity evaluation during 453 primer design covered only 259 non-target species.

454 In this work, 21 to 25 target strains for each target species and 120 non-target strains have been 455 tested to assess inclusivity and exclusivity of the qPCR assays, respectively. The in vitro 456 validation of primer pairs has shown that the in silico validation is not always able to predict 457 non-target PCR products. The fluorescence signals occurring at late quantification cycles $(\mathrm{Cq}>$ 458 30) are probably due to PCR products with suboptimal primer binding. Testing the qPCR assays 459 in mixtures and communities could be interesting to assess if these PCR products also 460 accumulate in presence of target DNA. The specificity could be sufficient in mixtures due to 461 competition for the primers and the difference in primer binding and amplification efficiency.

462 For many research applications, qPCR assays with a low signal in negative samples are 463 acceptable, assuming that low-level signals can be distinguished from low concentrations of 464 target species DNA by the melting curve analysis (Ririe et al. 1997). Furthermore, for many 465 applications, the annealing temperature can be optimized by empirical determination of a 466 suitable annealing temperature and the primer concentration can be adjusted to improve the 467 specificity of the assay (Bio-Rad Laboratories 2019). We did not try to optimize our assays with these measures, because the aim was to design primers for high-throughput qPCR, requiring the exact same PCR conditions. For the tested qPCR conditions, the most specific qPCR assays were Ec_faeca_acuI (E.faecalis), Ec_faeci_cysS (E.faecium), Pd_acidi_asnS (P. acidilactici) and Pd_pento_nagK (P.pentosaceus). Further work will be necessary in order to make these qPCR assays fully operational for the quantification of bacteria in a complex system such as food. For instance, suitable qPCR standards should be designed and validated, so that the limit of detection of each assay can be determined (Forootan et al. 2017).

475 Primer-BLAST, fdp and RUCS allow designing primers for different applications, but demand experience and manual manipulations. Primer-BLAST designs primers and performs specificity checks, but requires a target sequence provided by the user. In the case of RUCS, manual manipulation and some experience is needed to prepare the positive and negative reference sets.

479 The same applies to fdp and the results from the comparison indicate that fdp has its strength in 480 the identification of strain-specific primer pairs and for subsets of the positive set as implied in 481 the name. The observed mismatches in the primer-binding region are probably due to the 482 alignment-free approach the pipeline uses. This seems to drastically increase the speed, but it is 
483 not taking into account the conservation of the target sequence and therefore mismatches, e.g.

484 due to single nucleotide polymorphisms (SNPs), can be found in the primer binding region. For a

485 large number of input assemblies, e.g. for E. faecalis (575), fdp requires distinctively more time

486 to run, which is a known issue caused by the cross-validation prediction step using PrimerSearch

487 (Pritchard et al. 2012).

488 Compared to primer-BLAST and RUCS, the task SpeciesPrimer performs is really specialized,

489 namely to design primers for species-specific sequences. In contrast, SpeciesPrimer requires no

490 previous knowledge about the input genome assemblies and no manual manipulation of

491 sequences has to be performed. The ability of SpeciesPrimer to run on standard computers with

492 good performance instead of specialized high-performance computers should allow primer

493 design for the wider range of scientists. Docker containers simplify the installation procedure and

494 should allow non-bioinformaticians to setup and use the SpeciesPrimer pipeline.

\section{Conclusions}

496 In this work, we presented the SpeciesPrimer pipeline, which is a fully automated pipeline from

497 the download of bacterial genomes, the identification of conserved species-specific core genes to

498 primer design and subsequent quality control of primer candidates. Primers for four bacterial

499 species were designed and validated and have shown to perform adequately under the same

500 qPCR conditions.

501 A standard computer with good performance, good quality genome assemblies, a local copy of

502 the nt BLAST database and a list of non-target bacterial species are the only requirements for

503 primer design with SpeciesPrimer. A complete image of a Linux OS with all dependencies and

504 the pipeline scripts is available from Dockerhub. To simplify primer design for users not familiar

505 with command line tools, a graphic user interface is provided in the latest version of

506 SpeciesPrimer. SpeciesPrimer facilitates efficient primer design for species-specific

507 quantification, paving the way for a fast and accurate quantitative investigation of microbial

508 communities.

509 Acknowledgements

510 We would like to thank Marco Meola and Remo Schmidt for critically reading the manuscript

511 and many helpful comments and Daniel Marzohl, Nadine Sidler, Elvira Wagner and Kotchanoot

512 Srikham for their valuable technical help. 


\section{References}

514 Almeida M, Hébert A, Abraham A-L, Rasmussen S, Monnet C, Pons N, Delbès C, Loux V,

515

516

517

518

519

520

521

522

523

524

525

526

527

528

529

530

531

532

533

534

535

536

537

538

539

540

541

542

543

544

545

546

547

548

549

550

551

552

553

554

555

556

557

558
Batto J-M, Leonard P, Kennedy S, Ehrlich SD, Pop M, Montel M-C, Irlinger F, and Renault P. 2014. Construction of a dairy microbial genome catalog opens new perspectives for the metagenomic analysis of dairy fermented products. BMC Genomics 15:1101. 10.1186/1471-2164-15-1101

Altschul SF, Gish W, Miller W, Myers EW, and Lipman DJ. 1990. Basic local alignment search tool. J Mol Biol 215:403-410. 10.1016/s0022-2836(05)80360-2

Ciufo S, Kannan S, Sharma S, Badretdin A, Clark K, Turner S, Brover S, Schoch CL, Kimchi A, and DiCuccio M. 2018. Using average nucleotide identity to improve taxonomic assignments in prokaryotic genomes at the NCBI. Int J Syst Evol Microbiol 68:23862392. 10.1099/ijsem.0.002809

Cock PJ, Antao T, Chang JT, Chapman BA, Cox CJ, Dalke A, Friedberg I, Hamelryck T, Kauff F, Wilczynski B, and de Hoon MJ. 2009. Biopython: freely available Python tools for computational molecular biology and bioinformatics. Bioinformatics 25:1422-1423. 10.1093/bioinformatics/btp163

Cremonesi P, Pisani LF, Lecchi C, Ceciliani F, Martino P, Bonastre AS, Karus A, Balzaretti C, and Castiglioni B. 2014. Development of 23 individual TaqMan(R) real-time PCR assays for identifying common foodborne pathogens using a single set of amplification conditions. Food Microbiol 43:35-40. 10.1016/j.fm.2014.04.007

Curran T, Coyle PV, McManus TE, Kidney J, and Coulter WA. 2007. Evaluation of real-time PCR for the detection and quantification of bacteria in chronic obstructive pulmonary disease. FEMS Immunol Med Microbiol 50:112-118. 10.1111/j.1574-695X.2007.00241.x

Elbrecht V, Leese F, and Bunce M. 2017. PrimerMiner: an R package for development and in silico validation of DNA metabarcoding primers. Methods in Ecology and Evolution 8:622-626. 10.1111/2041-210x.12687

Falentin H, Postollec F, Parayre S, Henaff N, Le Bivic P, Richoux R, Thierry A, and Sohier D. 2010. Specific metabolic activity of ripening bacteria quantified by real-time reverse transcription PCR throughout Emmental cheese manufacture. Int J Food Microbiol 144:10-19. 10.1016/j.ijfoodmicro.2010.06.003

Forootan A, Sjoback R, Bjorkman J, Sjogreen B, Linz L, and Kubista M. 2017. Methods to determine limit of detection and limit of quantification in quantitative real-time PCR (qPCR). Biomol Detect Quantif 12:1-6. 10.1016/j.bdq.2017.04.001

Garrido-Maestu A, Azinheiro S, Carvalho J, and Prado M. 2018. Rapid and sensitive detection of viable Listeria monocytogenes in food products by a filtration-based protocol and qPCR. Food Microbiol 73:254-263. 10.1016/j.fm.2018.02.004

Hermann-Bank ML, Skovgaard K, Stockmarr A, Larsen N, and Mølbak L. 2013. The Gut Microbiotassay: a high-throughput qPCR approach combinable with next generation sequencing to study gut microbial diversity. BMC Genomics 14:788. 10.1186/1471-216414-788

Ishii S, Segawa T, and Okabe S. 2013. Simultaneous Quantification of Multiple Food- and Waterborne Pathogens by Use of Microfluidic Quantitative PCR. Appl Environ Microbiol 79:2891-2898. 10.1128/aem.00205-13

Kim M, Oh HS, Park SC, and Chun J. 2014. Towards a taxonomic coherence between average nucleotide identity and 16S rRNA gene sequence similarity for species demarcation of prokaryotes. Int J Syst Evol Microbiol 64:346-351 . 10.1099/ijs.0.059774-0

PeerJ reviewing PDF | (2019:07:39105:2:0:NEW 19 Dec 2019) 
559

560

561

562

563

564

565

566

567

568

569

570

571

572

573

574

575

576

577

578

579

580

581

582

583

584

585

586

587

588

589

590

591

592

593

594

595

596

597

598

599

600

601

602

603
Kleyer H, Tecon R, and Or D. 2017. Resolving Species Level Changes in a Representative Soil Bacterial Community Using Microfluidic Quantitative PCR. Frontiers in Microbiology 8. 10.3389/fmicb.2017.02017

Masco L, Vanhoutte T, Temmerman R, Swings J, and Huys G. 2007. Evaluation of real-time PCR targeting the 16S rRNA and recA genes for the enumeration of bifidobacteria in probiotic products. International Journal of Food Microbiology 113:351-357.

http://dx.doi.org/10.1016/j.ijfoodmicro.2006.07.021

Moyaert H, Pasmans F, Ducatelle R, Haesebrouck F, and Baele M. 2008. Evaluation of 16S rRNA Gene-Based PCR Assays for Genus-Level Identification of Helicobacter Species. $J$ Clin Microbiol 46:1867-1869. 10.1128/jcm.00139-08

Page AJ, Cummins CA, Hunt M, Wong VK, Reuter S, Holden MT, Fookes M, Falush D, Keane JA, and Parkhill J. 2015. Roary: rapid large-scale prokaryote pan genome analysis. Bioinformatics 31:3691-3693. 10.1093/bioinformatics/btv421

Postollec F, Falentin H, Pavan S, Combrisson J, and Sohier D. 2011. Recent advances in quantitative PCR (qPCR) applications in food microbiology. Food Microbiol 28:848-861. http://dx.doi.org/10.1016/j.fm.2011.02.008

Pritchard L, Glover RH, Humphris S, Elphinstone JG, and Toth IK. 2016. Genomics and taxonomy in diagnostics for food security: soft-rotting enterobacterial plant pathogens. Analytical Methods 8:12-24. 10.1039/c5ay02550h

Pritchard L, Holden NJ, Bielaszewska M, Karch H, and Toth IK. 2012. Alignment-free design of highly discriminatory diagnostic primer sets for Escherichia coli O104:H4 outbreak strains. PLoS One 7:e34498. 10.1371/journal.pone.0034498

Qu W, Zhou Y, Zhang Y, Lu Y, Wang X, Zhao D, Yang Y, and Zhang C. 2012. MFEprimer-2.0: a fast thermodynamics-based program for checking PCR primer specificity. Nucleic Acids Res 40:W205-208. 10.1093/nar/gks552

Ramirez M, Castro C, Palomares JC, Torres MJ, Aller AI, Ruiz M, Aznar J, and MartinMazuelos E. 2009. Molecular detection and identification of Aspergillus spp. from clinical samples using real-time PCR. Mycoses 52:129-134. 10.1111/j.14390507.2008.01548.x

Rice P, Longden I, and Bleasby A. 2000. EMBOSS: The European Molecular Biology Open Software Suite. Trends in Genetics 16:276-277. https://doi.org/10.1016/S01689525(00)02024-2

Richter M, and Rossello-Mora R. 2009. Shifting the genomic gold standard for the prokaryotic species definition. Proc Natl Acad Sci U S A 106:19126-19131. 10.1073/pnas.0906412106

Ririe KM, Rasmussen RP, and Wittwer CT. 1997. Product Differentiation by Analysis of DNA Melting Curves during the Polymerase Chain Reaction. Analytical Biochemistry 245:154160. https://doi.org/10.1006/abio.1996.9916

Scheirlinck I, Van der Meulen R, De Vuyst L, Vandamme P, and Huys G. 2009. Molecular source tracking of predominant lactic acid bacteria in traditional Belgian sourdoughs and their production environments. J Appl Microbiol 106:1081-1092. 10.1111/j.13652672.2008.04094.x

Shen Z, Qu W, Wang W, Lu Y, Wu Y, Li Z, Hang X, Wang X, Zhao D, and Zhang C. 2010. MPprimer: a program for reliable multiplex PCR primer design. BMC Bioinformatics 11:143. 10.1186/1471-2105-11-143 
604 Tange O. 2011. GNU Parallel - The Command-Line Power Tool. login: The USENIX Magazine $605 \quad 36: 42-47$.

606 Tao T, Madden T, Christiam C, and Szilagyi L. 2011. BLAST FTP Site. Available at

607 https://www.ncbi.nlm.nih.gov/books/NBK62345/(accessed September 242019$).$

608 Thomsen MCF, Hasman H, Westh H, Kaya H, and Lund O. 2017. RUCS: rapid identification of

$609 \quad$ PCR primers for unique core sequences. Bioinformatics 33:3917-3921.

$610 \quad$ 10.1093/bioinformatics/btx526

611 Torriani S, Felis GE, and Dellaglio F. 2001. Differentiation of Lactobacillus plantarum, L.

612 pentosus, and L. paraplantarum by recA gene sequence analysis and multiplex PCR assay

613 with recA gene-derived primers. Appl Environ Microbiol 67:3450-3454.

$614 \quad 10.1128$ /aem.67.8.3450-3454.2001

615 Untergasser A, Cutcutache I, Koressaar T, Ye J, Faircloth BC, Remm M, and Rozen SG. 2012.

616 Primer3--new capabilities and interfaces. Nucleic Acids Res 40:e115. 10.1093/nar/gks596

617 Vijaya Satya R, Kumar K, Zavaljevski N, and Reifman J. 2010. A high-throughput pipeline for

618 the design of real-time PCR signatures. BMC Bioinformatics 11:340. 10.1186/1471-2105-

$619 \quad 11-340$

620

621

622

623

624

625

626

627

628

629

630

631

Wang LT, Lee FL, Tai CJ, and Kasai H. 2007. Comparison of gyrB gene sequences, 16S rRNA gene sequences and DNA-DNA hybridization in the Bacillus subtilis group. Int $J$ Syst Evol Microbiol 57:1846-1850. 10.1099/ijs.0.64685-0

Ye J, Coulouris G, Zaretskaya I, Cutcutache I, Rozen S, and Madden TL. 2012. Primer-BLAST: a tool to design target-specific primers for polymerase chain reaction. $B M C$ Bioinformatics 13:134. 10.1186/1471-2105-13-134

Zhu T, Liang C, Meng Z, Li Y, Wu Y, Guo S, and Zhang R. 2017. PrimerServer: a highthroughput primer design and specificity-checking platform. bioRxiv:181941. $10.1101 / 181941$

Zuker M, Mathews DH, and Turner DH. 1999. Algorithms and Thermodynamics for RNA Secondary Structure Prediction: A Practical Guide. In: Barciszewski J, and Clark BFC, eds. RNA Biochemistry and Biotechnology. Dordrecht: Springer Netherlands, 11-43.

632

633 


\section{Table $\mathbf{1}$ (on next page)}

Overview of the features of different primer design tools and pipelines. 


\begin{tabular}{|c|c|c|c|c|c|c|c|}
\hline Tool & RUCS & fdp & TOPSI & $\begin{array}{l}\text { Species- } \\
\text { Primer }\end{array}$ & $\begin{array}{l}\text { Primer- } \\
\text { Miner }\end{array}$ & $\begin{array}{l}\text { Primer- } \\
\text { Server }\end{array}$ & $\begin{array}{l}\text { Primer- } \\
\text { BLAST }\end{array}$ \\
\hline Reference & $\begin{array}{l}\text { (Thomsen et } \\
\text { al. 2017) }\end{array}$ & $\begin{array}{l}\text { (Pritchard } \\
\text { et al. 2012) }\end{array}$ & $\begin{array}{c}\text { (Vijaya } \\
\text { Satya et al. } \\
\text { 2010) }\end{array}$ & (this study) & $\begin{array}{l}\text { (Elbrecht et } \\
\text { al. 2017) }\end{array}$ & $\begin{array}{c}\text { (Zhu et al. } \\
\text { 2017) }\end{array}$ & $\begin{array}{c}\text { (Ye et al. } \\
\text { 2012) }\end{array}$ \\
\hline Primer specificity & \multicolumn{2}{|c|}{ Bacterial strains / species } & \multicolumn{2}{|c|}{ Bacterial species } & $\begin{array}{l}\text { Taxonomic } \\
\text { groups }\end{array}$ & \multicolumn{2}{|c|}{ Input sequence } \\
\hline \multicolumn{8}{|l|}{ Inputs } \\
\hline Taxonomic group(s) & - & - & - & Species & $\begin{array}{l}\text { Order, } \\
\text { Family }\end{array}$ & - & - \\
\hline Target gene(s) & - & - & - & - & $\mathrm{x}$ & - & $\mathrm{x}$ \\
\hline Genome assemblies & $\mathrm{x}$ & $\mathrm{x}$ & $\mathrm{x}$ & $\mathrm{x}$ & - & - & - \\
\hline Target sequences & - & - & - & - & $\mathrm{x}$ & $\mathrm{x}$ & $\mathrm{x}$ \\
\hline Primer sequences & $\mathrm{x}$ & $\mathrm{x}$ & - & - & $\mathrm{x}$ & $\mathrm{x}$ & $\mathrm{x}$ \\
\hline $\begin{array}{l}\text { Automatic download } \\
\text { of input sequences }\end{array}$ & - & - & - & $\mathrm{x}$ & $\mathrm{x}$ & - & - \\
\hline $\begin{array}{l}\text { Identification of } \\
\text { target sequences }\end{array}$ & $\mathrm{x}$ & $\mathrm{x}$ & $\mathrm{x}$ & $\mathrm{x}$ & - & - & - \\
\hline $\begin{array}{l}\text { Identification of } \\
\text { conserved regions }\end{array}$ & $\mathrm{x}$ & - & $\mathrm{x}$ & $\mathrm{x}$ & $\mathrm{x}$ & - & - \\
\hline Primer design & $\mathrm{x}$ & $\mathrm{x}$ & $\mathrm{x}$ & $\mathrm{x}$ & - & $\mathrm{x}$ & $\mathrm{x}$ \\
\hline \multicolumn{8}{|l|}{ Specificity check } \\
\hline Target sequences & $\begin{array}{c}\text { Input } \\
\text { sequences }\end{array}$ & $\begin{array}{l}\text { Input } \\
\text { sequences }\end{array}$ & BLAST DB & BLAST DB & - & - & BLAST DB \\
\hline Primer & $\begin{array}{c}\text { Input } \\
\text { sequences }\end{array}$ & BLAST DB & BLAST DB & BLAST DB & Alignment & BLAST DB & BLAST DB \\
\hline $\begin{array}{l}\text { Primer quality } \\
\text { control }\end{array}$ & $\mathrm{x}$ & $\mathrm{x}$ & $\mathrm{x}$ & $\mathrm{x}$ & - & $\mathrm{x}$ & \\
\hline Primer 3 cutoffs & $\mathrm{x}$ & $\mathrm{x}$ & $\mathrm{x}$ & $\mathrm{x}$ & - & $\mathrm{x}$ & $\mathrm{x}$ \\
\hline Primer dimer & - & - & - & $\mathrm{x}$ & - & - & - \\
\hline Hairpin & - & - & - & - & - & - & - \\
\hline $\begin{array}{l}\text { Amplicon } \\
\text { secondary structures }\end{array}$ & - & - & - & $\mathrm{x}$ & - & - & - \\
\hline $\begin{array}{l}\text { High-throughput } \\
\text { primer design }\end{array}$ & $\mathrm{x}$ & $\mathrm{x}$ & $\mathrm{x}$ & $\mathrm{x}$ & - & $\mathrm{x}$ & - \\
\hline Batch processing & - & - & - & Full runs & Download & - & - \\
\hline $\begin{array}{l}\text { Works on standard } \\
\text { computers }\end{array}$ & $\mathrm{x}$ & $\mathrm{x}$ & - & $\mathrm{x}$ & $\mathrm{x}$ & $\mathrm{x}$ & - \\
\hline $\begin{array}{l}\text { Graphic user } \\
\text { interface }\end{array}$ & $\mathrm{x}$ & - & - & $\mathrm{x}$ & - & $\mathrm{x}$ & $\mathrm{x}$ \\
\hline Web service & $\mathrm{x}$ & - & * & - & - & $\mathrm{x}$ & $\mathrm{x}$ \\
\hline
\end{tabular}

1 fdp: find_differential_primers

2 x: Feature supported

3 - : Feature not supported

4 *: Access has to be requested

5 QC: Quality control

6 CDS: Coding sequences 
Table 2 (on next page)

Overview of the SpeciesPrimer pipeline workflow and the used software. 


\begin{tabular}{|c|c|c|}
\hline Pipeline workflow & Tools (Version*) & Reference \\
\hline $\begin{array}{l}\text { Input genome assemblies } \\
\text { - download } \\
\text { - annotation } \\
\text { - quality control }\end{array}$ & $\begin{array}{l}\text { NCBI Entrez (Biopython 1.73) } \\
\text { Prokka }(1.13 .7) \\
\text { BLAST+ }(2.9 .0+)\end{array}$ & $\begin{array}{l}\text { (Cock et al. 2009; Sayers 2009) } \\
\text { (Seemann 2014) } \\
\text { (Altschul et al. 1990) }\end{array}$ \\
\hline $\begin{array}{l}\text { Core gene sequences } \\
\text { - identification } \\
\text { - phylogeny } \\
\text { - selection of conserved } \\
\text { sequences } \\
\text { - evaluation of specificity }\end{array}$ & $\begin{array}{l}\text { Roary }(3.12 .0) \\
\text { FastTree } 2(2.1 .11) \\
\text { Prank (.150803) } \\
\text { consambig (EMBOSS 6.6.0.0) } \\
\text { GNU parallel (20161222) } \\
\text { BLAST+ }\end{array}$ & $\begin{array}{l}\text { (Page et al. 2015) } \\
\text { (Price et al. 2010) } \\
\text { (Löytynoja 2014) } \\
\text { (Rice et al. 2000) } \\
\text { (Tange 2011) } \\
\text { (Altschul et al. 1990) }\end{array}$ \\
\hline $\begin{array}{l}\text { Primer } \\
\qquad \begin{array}{l}\text { - design } \\
\text { - quality control }\end{array}\end{array}$ & $\begin{array}{l}\text { Primer3 (2.4.0) } \\
\text { BLAST+, } \\
\text { MFEprimer (2.0), } \\
\text { MPprimer (1.5), } \\
\text { Mfold (3.6) }\end{array}$ & $\begin{array}{l}\text { (Untergasser et al. 2012) } \\
\text { (Altschul et al. 1990) } \\
\text { (Qu et al. 2012) } \\
\text { (Shen et al. 2010) } \\
\text { (Zuker et al. 1999) }\end{array}$ \\
\hline
\end{tabular}

* Docker image June 13, 2019 


\section{Table 3 (on next page)}

Pipeline input and run statistics.

Two different computers were used to run the SpeciesPrimer pipeline depicted as high end desktop and consumer laptop. The high end desktop is running Ubuntu 16.04 in a virtual machine with two Xeon E5-2643 CPU's (22 logical processors), 32 GB RAM and a solid-state drive. The download of the genome assemblies was performed using a LAN connection. The consumer laptop is running on Ubuntu 16.04 with an i7-3610QM CPU (8 logical processors), 8 GB RAM and a solid-state drive. The download of the genome assemblies was performed using a wireless LAN connection. 


\begin{tabular}{|lcccc|}
\hline Species & E. faecalis & E. faecium & P. acidilactici & P. pentosaceus \\
\hline Pipeline input & & & & \\
NCBI genomes & 390 & 575 & 9 & 14 \\
ACC genomes & 0 & 0 & 118 & 13 \\
Total genome assemblies & $\mathbf{3 9 0}$ & $\mathbf{5 7 5}$ & $\mathbf{1 2 7}$ & $\mathbf{2 7}$ \\
\hline Download and annotation & & & & \\
(h:min) & & & & \\
High end desktop & $9: 04$ & $12: 27$ & $1: 55$ & $0: 24$ \\
Consumer laptop & $15: 56$ & $22: 18$ & $3: 10$ & $0: 42$ \\
Pipeline statistics & & & & \\
Running time (h:min) & & & & \\
High end desktop & $6: 11$ & $8: 05$ & $1: 55$ & $4: 25$ \\
Consumer laptop & $19: 52$ & $28: 56$ & $6: 59$ & $6: 47$ \\
Core genes & 1375 & 1131 & 921 & 1341 \\
Single copy core genes & 632 & 563 & 641 & 889 \\
Conserved sequences & 1128 & 624 & 566 & 2782 \\
Species-specific sequences & 329 & 36 & 54 & 672 \\
Potential primer pairs & 89 & 4 & 7 & 632 \\
Primer pairs after QC & $\mathbf{1 5}$ & $\mathbf{2}$ & $\mathbf{2}$ & $\mathbf{1 6 0}$ \\
\hline
\end{tabular}

1 QC: primer quality control, ACC: Agroscope Culture Collection 


\section{Table 4(on next page)}

Summary of the in silico validation of the selected primer pairs.

Primer pair coverage (PPC) is a value used by MFEprimer-2.0 to score the ability of the primer pair to bind to a DNA template. The number of perfect matches of the primers to the primer binding region and the total number of target sequences are indicated in brackets. 


\begin{tabular}{|l|l|l|l|l|}
\hline Target species & Primer pair & PPC & $\begin{array}{l}\text { BLAST } \\
\text { (perfect/total) }\end{array}$ & $\begin{array}{l}\text { Primer-BLAST } \\
\text { (perfect/total) }\end{array}$ \\
\hline \multirow{3}{*}{. faecalis } & Ec_faeca_acuI_1_P0 & 100 & specific $(694 / 694)$ & specific $(55 / 55)$ \\
\cline { 2 - 5 } & Ec_faeca_g3060_1_P0 & 100 & specific $(689 / 690)$ & specific $(55 / 55)$ \\
\hline \multirow{3}{*}{. faecium } & Ec_faeci_cysS_3_P1 & 96.7 & specific $(1057 / 1058)$ & specific $(148 / 148)$ \\
\cline { 2 - 5 } & Ec_faeci_purD_2_P0 & 93.3 & specific $(1083 / 1083)$ & specific $(148 / 148)$ \\
\hline \multirow{3}{*}{. acidilactici } & Pd_acidi_asnS_2_P0 & 90.1 & specific $(19 / 19)$ & specific $(11 / 11)$ \\
\cline { 2 - 5 } & Pd_acidi_g1164_1_P0 & 93.3 & specific $(23 / 23)$ & specific $(11 / 11)$ \\
\hline \multirow{3}{*}{ P. pentosaceus } & Pd_pento_nagK_1_P0 & 100 & specific $(15 / 15)$ & specific $(9 / 9)$ \\
\cline { 2 - 5 } & Pd_pento_g4364_1_P0 & 100 & specific $(15 / 15)$ & specific $(9 / 9)$ \\
\hline
\end{tabular}




\section{Table 5 (on next page)}

Summarized results of the in vitro validation of the selected qPCR assays.

Inclusivity: Number of positive DNA samples / total number of target species DNA samples. Exclusivity: Number of DNA samples showing a fluorescence signal below quantification cycle 35 and a melting curve peak above the threshold / total number of non-target DNA samples.

Calculated efficiency, slope, intercept and correlation coefficient $\left(R^{2}\right)$ of the linear regression equation. 


\begin{tabular}{|l|c|c|c|c|c|c|c|c|}
\hline Species & \multicolumn{2}{|c|}{ E. faecalis } & \multicolumn{2}{c|}{ E. faecium } & \multicolumn{2}{c|}{$P$. acidilactici } & \multicolumn{2}{c|}{ P. pentosaceus } \\
\hline Target gene & acuI & $\mathrm{g} 3060$ & cys & purD & asnS & $\mathrm{g} 1164$ & $n a g K$ & $\mathrm{~g} 4364$ \\
\hline Inclusivity & $22 / 22$ & $22 / 22$ & $25 / 25$ & $24 / 25$ & $21 / 21$ & $21 / 21$ & $25 / 25$ & $25 / 25$ \\
\hline Exclusivity & $0 / 120$ & $9 / 120$ & $0 / 120$ & $0 / 120$ & $0 / 120$ & $5 / 120$ & $2 / 120$ & $8 / 120$ \\
\hline Efficiency & $98 \%$ & $97 \%$ & $92 \%$ & $97 \%$ & $99 \%$ & $100 \%$ & $94 \%$ & $92 \%$ \\
\hline Slope & -3.382 & -3.387 & -3.539 & -3.396 & -3.356 & -3.329 & -3.470 & -3.523 \\
\hline Intercept & 32.107 & 32.694 & 32.006 & 31.051 & 30.835 & 30.282 & 32.286 & 33.211 \\
\hline $\mathbf{R}^{\mathbf{2}}$ & 0.998 & 0.997 & 0.990 & 0.996 & 0.997 & 0.995 & 0.996 & 0.997 \\
\hline
\end{tabular}

1 


\section{Table 6(on next page)}

Comparison of different primer design pipelines.

The hardware used to run the pipelines was a high end desktop running Ubuntu 16.04 in a virtual machine with two Xeon E5-2643 CPU's (22 logical processors), 32 GB RAM and a solidstate drive. For the Primer-BLAST results the number of perfect matches of the primers to the primer binding region and the total number of target sequences are indicated in brackets. 


\begin{tabular}{|c|c|c|c|c|}
\hline Target species & Enterococcus faecalis & Enterococcus faecium & Pediococcus acidilactici & Pediococcus pentosaceus \\
\hline \multicolumn{5}{|c|}{ Running time (h:min) } \\
\hline RUCS & $5: 17$ & $5: 20$ & $2: 11$ & $3: 16$ \\
\hline fdp & $8: 32$ & $17: 44$ & $0: 36$ & $0: 02$ \\
\hline SpeciesPrimer & $5: 23$ & $6: 53$ & $1: 35$ & $1: 11$ \\
\hline \multicolumn{5}{|l|}{ Primer pairs } \\
\hline RUCS & 199 & 107 & 123 & 629 \\
\hline $\mathrm{fdp}$ & 6 & 3 & 3 & 6 \\
\hline SpeciesPrimer & 11 & 3 & 2 & 36 \\
\hline \multicolumn{5}{|c|}{ Primer-BLAST (perfect / total) } \\
\hline \multicolumn{5}{|l|}{ Best primer pair } \\
\hline fdp & specific $(58 / 59)$ & specific $(153 / 153)$ & specific $(10 / 11)$ & specific $(9 / 9)$ \\
\hline SpeciesPrimer & specific $(59 / 59)$ & specific $(153 / 153)$ & specific $(11 / 11)$ & specific $(9 / 9)$ \\
\hline
\end{tabular}

1 fdp: find_differential_primers 
Figure 1

Schematic workflow of the database creation and the specificity check using MFEprimer-2.0.

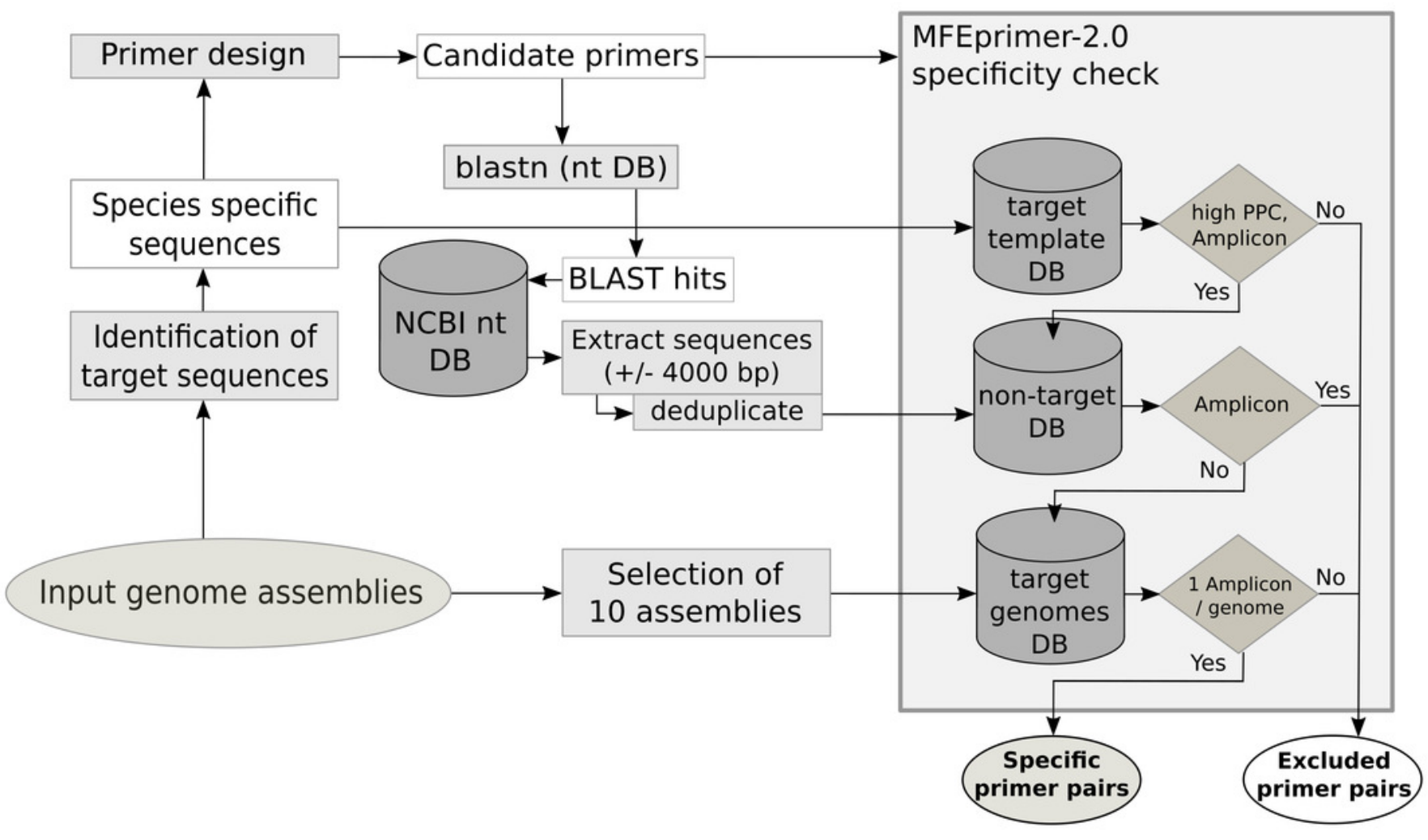


Figure 2

Phylogenetic tree based on the alignment of concatenated core genes of 575 Enterococcus faecium genome assemblies.

(A) The main cluster with 531 sequences is depicted in black and the subcluster of 44 sequences in blue. (B) Enlarged view of the tree structure and the subcluster. 
A

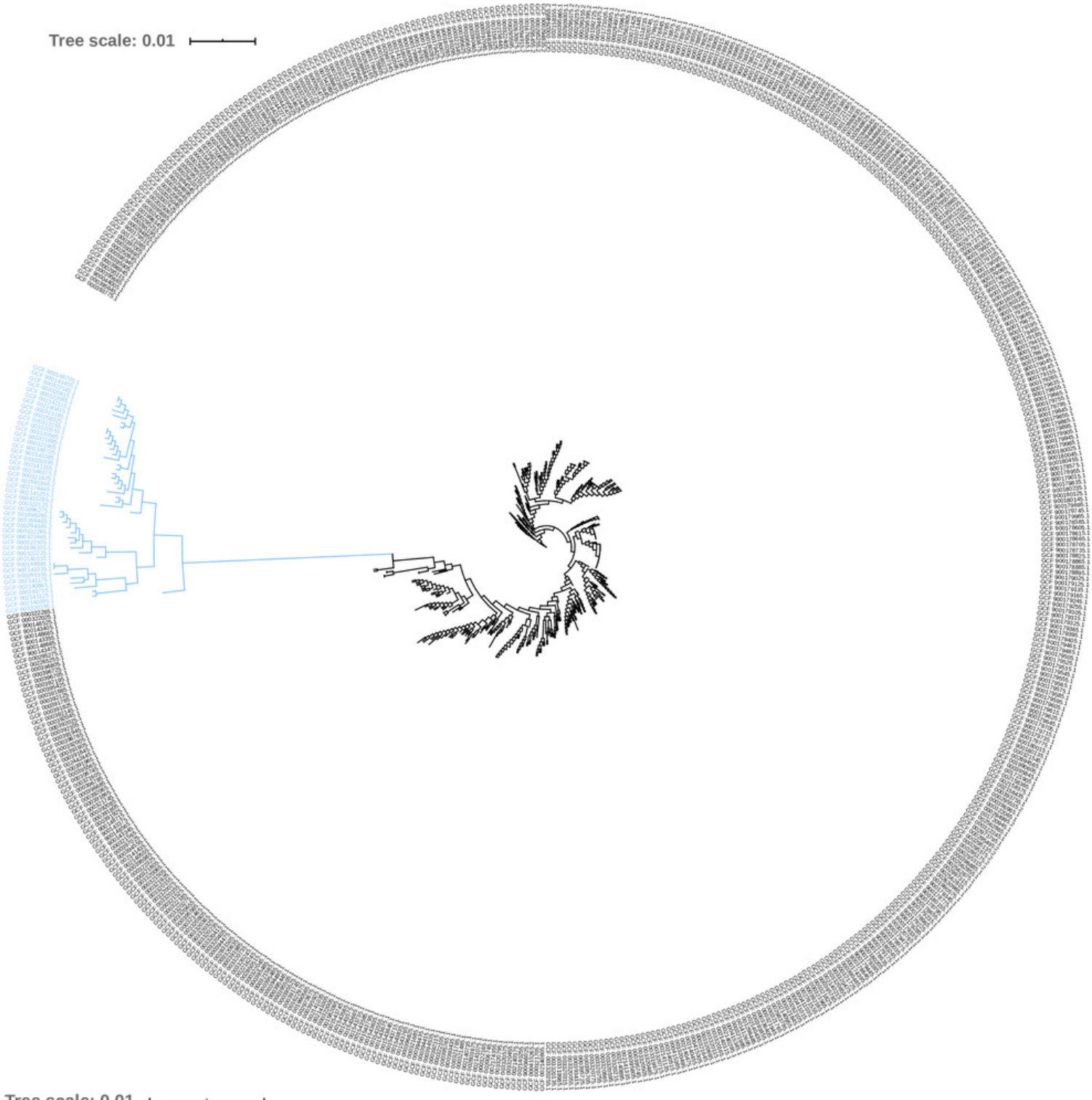

B

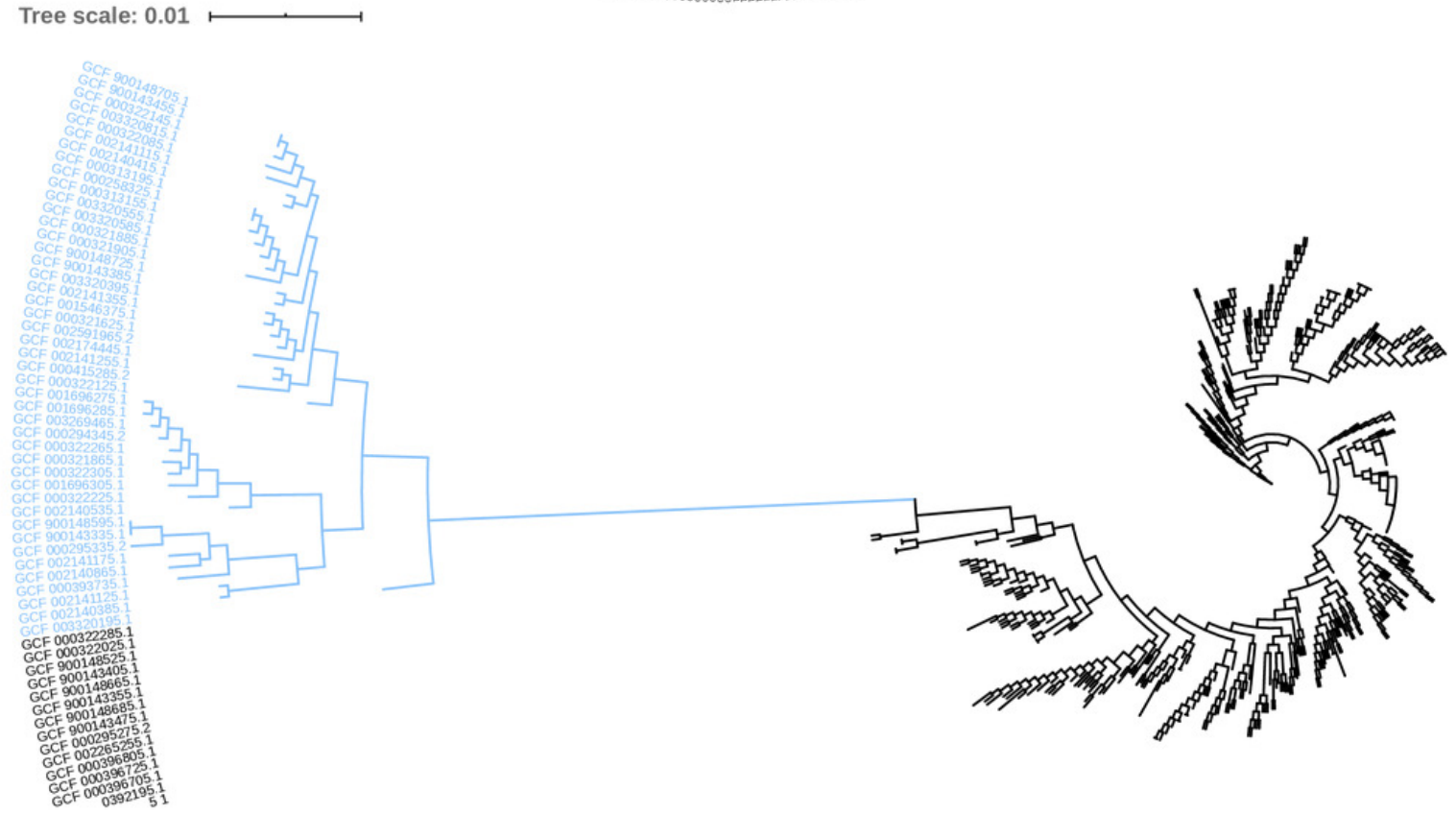

Peer) reviewing PDF | (2019:07:39105:2:0:NEW 19 Dec 2019) 
Figure 3

Phylogenetic tree based on the alignment of concatenated core genes of 127 Pediococcus acidilactici genome assemblies.

The main cluster with 100 sequences is depicted in black, a subcluster of 26 sequences in blue and the sequence with the largest phylogenetic distance in orange. 
Tree scale: $0.01 \longmapsto$

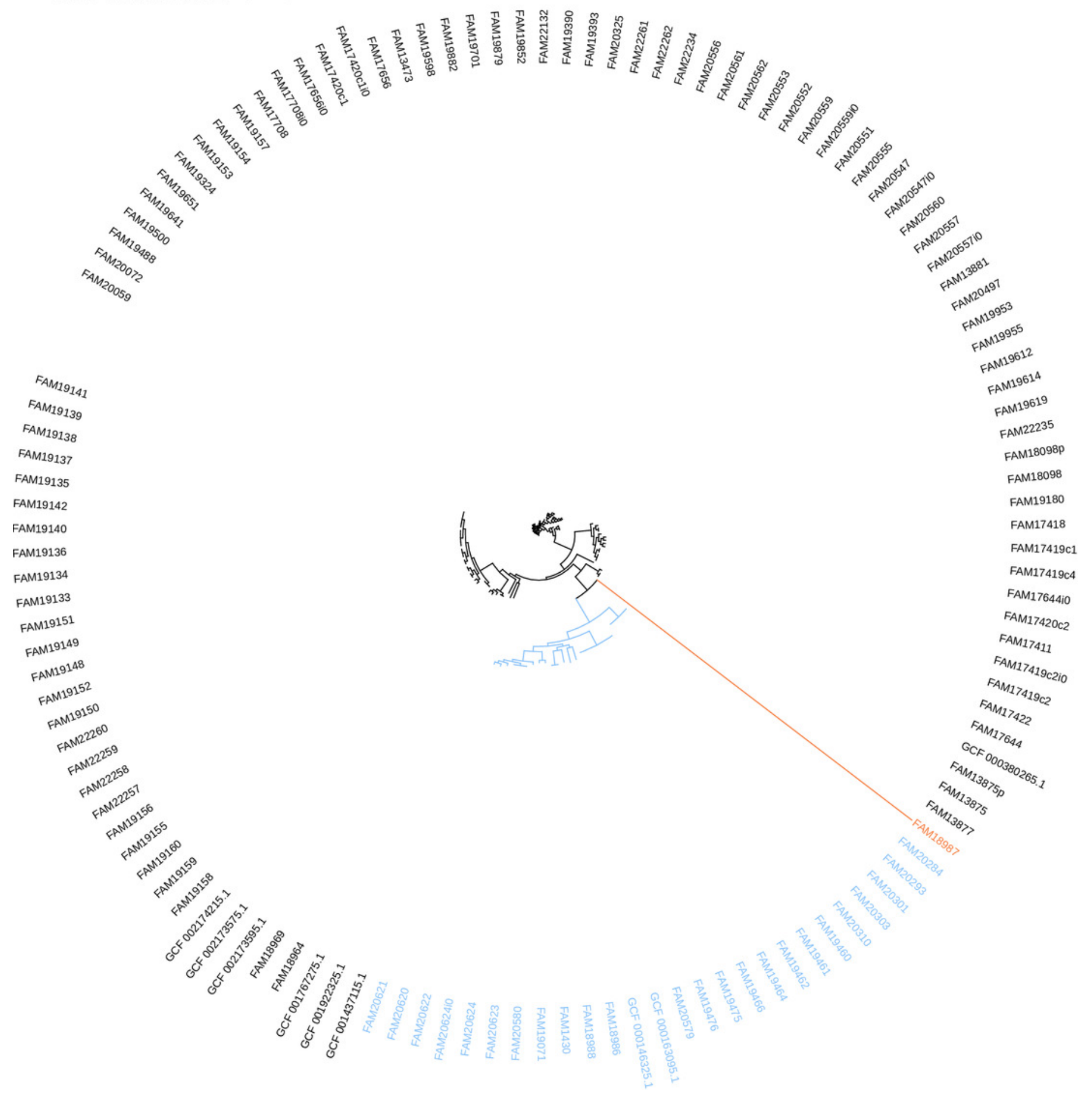




\section{Figure 4}

Clustered heatmap of ANIm percentage identity for (A) 575 Enterococcus faecium and (B) 129 Pediococcus acidilactici genome assemblies.

Purple colored cells in the heatmap correspond to ANIm percentage identity above $95 \%$, color intensity fades towards the proposed species threshold cutoff. Blue colored cells are below this threshold indicating that the corresponding genome assembly does not belong to the same species. Color bars on top and on the left of the heatmap correspond to the clusters and the colors indicated in the phylogenetic trees (Figure 2 and 3). Dendograms are based on single-linkage hierarchical clustering of the ANIm percentage identities. Row and column names can be found in Dataset S3.
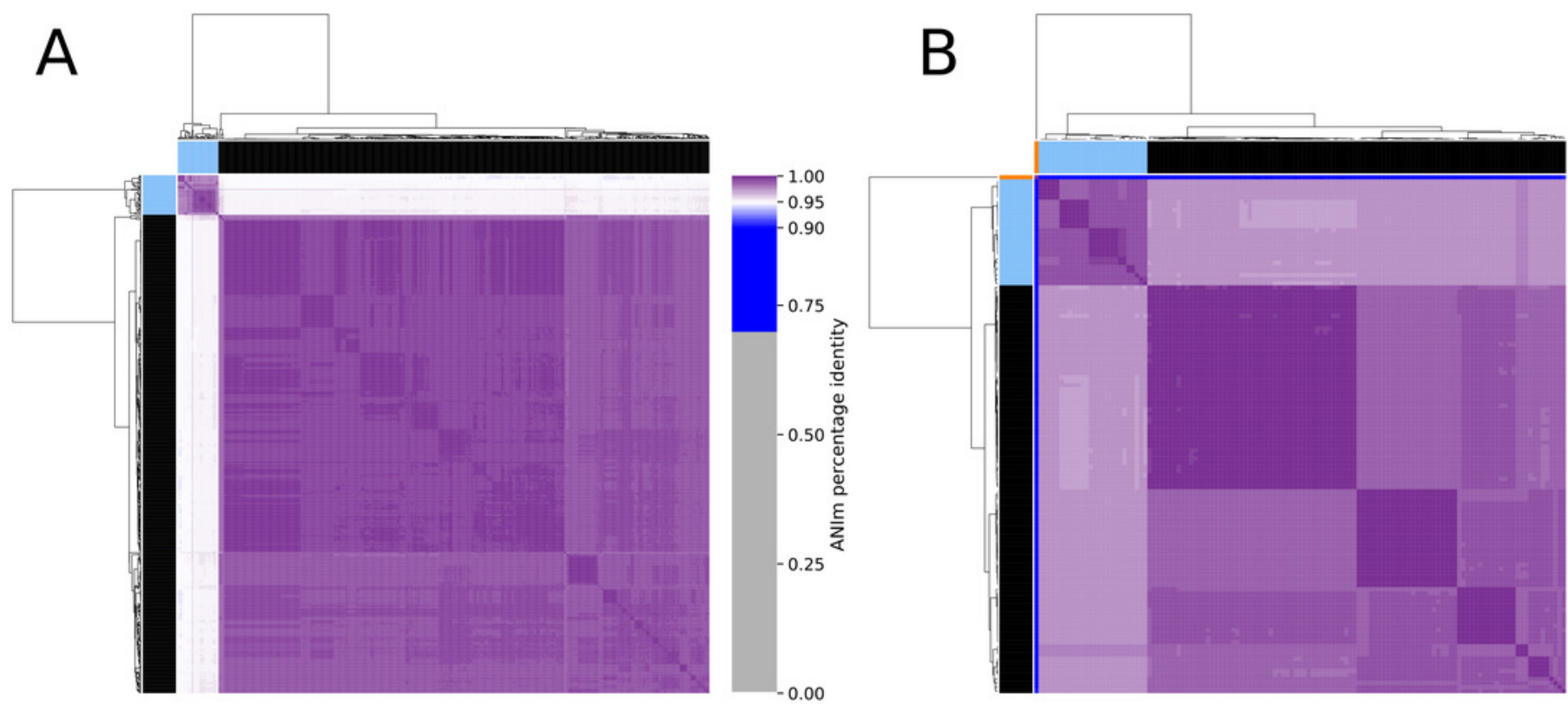


\section{Figure 5}

qPCR assay quantification cycle heatmap.

Depicted are all tested non-target strains and their average quantification cycle (technical duplicates) if the melt curve peak was above the threshold. The gray shades represent the Cq values from 10 to 35 (if no fluorescent signal was measured the value was set to $\mathrm{Cq} 35$ ). Abbreviations: A.: Acidipropionici; Cl.: Clostridium; Lb.: Lactobacillus; Ln.: Leuconostoc; Pb: Propionibacterium; Pd.: Pediococcus; Sc.: Streptococcus; NTC: no template control. 


\section{Species}

\begin{tabular}{|c|c|}
\hline$=$ & A.acidipropionici \\
\hline 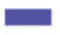 & Cl.beijerinckii \\
\hline 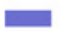 & Cl.butyricum \\
\hline 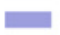 & Cl.ljungdahlii \\
\hline 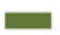 & Cl.sporogenes \\
\hline 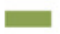 & Cl.tyrobutyricum \\
\hline 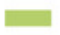 & Ec.durans \\
\hline 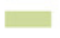 & Ec.faecalis \\
\hline 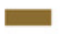 & Ec.faecium \\
\hline 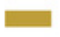 & Lb.acidophilus \\
\hline 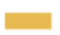 & Lb.amylovorus \\
\hline 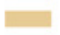 & Lb.brevis \\
\hline 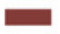 & Lb.buchneri \\
\hline ש & Lb.casei \\
\hline 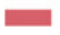 & Lb.coryniformis \\
\hline 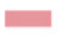 & Lb.coryniformis subsp. coryniformis \\
\hline$\square$ & Lb.coryniformis subsp. torquens \\
\hline 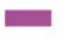 & Lb.crispatus \\
\hline$\square$ & Lb.curvatus \\
\hline$=$ & Lb.delbrueckii subsp. bulgaricus \\
\hline 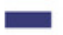 & Lb.delbrueckii subsp. delbrueckii \\
\hline - & Lb.delbrueckii subsp. lactis \\
\hline$=$ & Lb.equicursoris \\
\hline$\square$ & Lb.fermentum \\
\hline 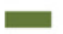 & Lb.gallinarum \\
\hline 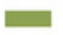 & Lb.graminis \\
\hline 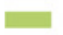 & Lb.helveticus \\
\hline 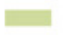 & Lb.kefiri \\
\hline 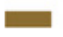 & Lb.koreensis \\
\hline 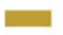 & Lb.parabuchneri \\
\hline 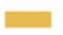 & Lb.paracasei \\
\hline$=$ & Lb.paraplantarum \\
\hline 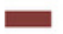 & Lb.pentosus \\
\hline 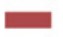 & Lb.plantarum \\
\hline$=$ & Lb.rhamnosus \\
\hline - & Lb.sakei \\
\hline - & Lb.sakei subsp. carnosum \\
\hline - & Lb.sakei subsp. sakei \\
\hline 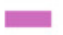 & Lb.zeae \\
\hline- & Lc.garvieae subsp. garvieae \\
\hline $\mathbf{0}$ & Lc.lactis subsp. cremoris \\
\hline 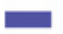 & Lc.lactis subsp. lactis \\
\hline $\mathbf{\omega}$ & Lc.lactis subsp. lactis biovar diacetylactis \\
\hline - & Ln.carnosum \\
\hline 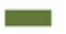 & Ln.lactis \\
\hline$=$ & Ln.mesenteroides subsp. cremoris \\
\hline 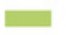 & Ln.mesenteroides subsp. dextranicum \\
\hline 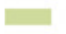 & Ln.mesenteroides subsp. mesenteroides \\
\hline - & Ln.mesenteroides subsp. suionicum \\
\hline 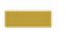 & Ln.pseudomesenteroides \\
\hline 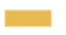 & NTC \\
\hline 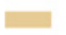 & $\mathrm{Pb}$.freudenreichii \\
\hline 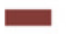 & Pd.acidilactici \\
\hline - & Pd.pentosaceus \\
\hline$\square$ & Pd.stilesii \\
\hline 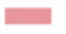 & Sc.salivarius \\
\hline - & Sc.salivarius subsp. salivarius \\
\hline & Sc.thermophilus \\
\hline
\end{tabular}
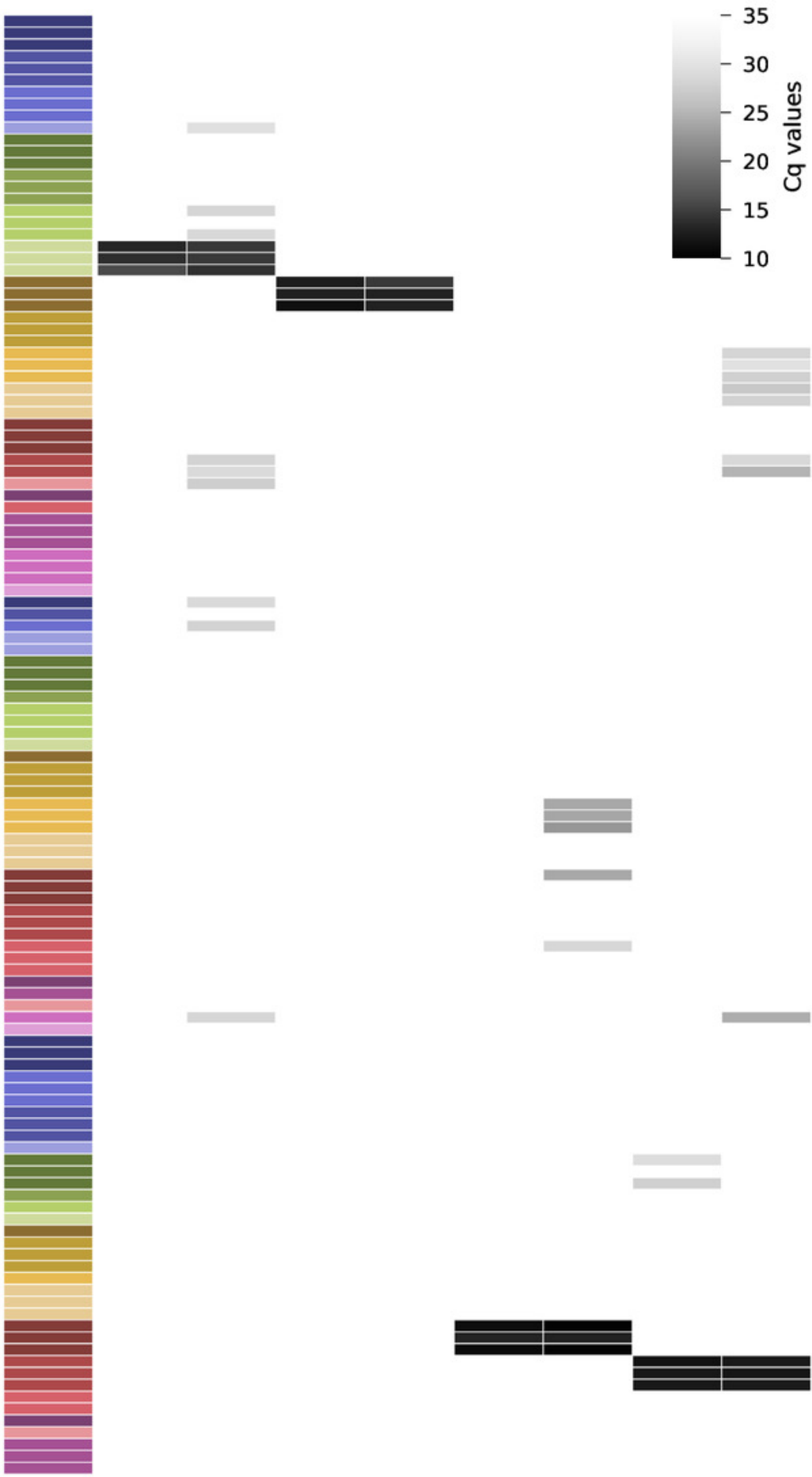

10

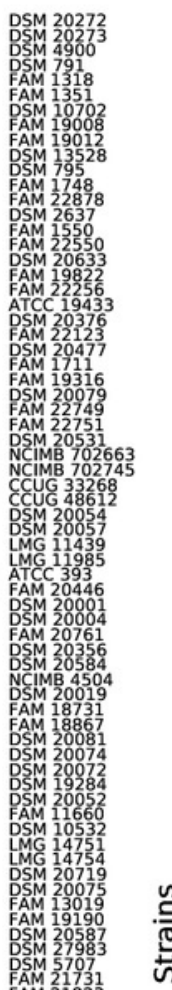

